Please do not remove this page

RMIT

UNIVERSITY

\title{
A mixed integer programming approach for asset protection during escaped wildfires
}

Van der Merwe, Martijn; Minas, James; Ozlen, Melih; Hearne, John

https://researchrepository.rmit.edu.au/esploro/outputs/9921862128401341/filesAndLinks?institution=61RMIT_INST\&index=null

Van der Merwe, M., Minas, J., Ozlen, M., \& Hearne, J. (2015). A mixed integer programming approach for asset protection during escaped wildfires. Canadian Journal of Forest Research, 45(4), 444-451.

https://doi.org/10.1139/cjfr-2014-0239

Document Version: Accepted Manuscript

Published Version: https://doi.org/10.1139/cjfr-2014-0239

Repository homepage: https://researchrepository.rmit.edu.au

(c) 2014, National Research Council of Canada.All rights reserved.

Downloaded On 2023/04/26 20:06:02 +1000 
Thank you for downloading this document from the RMIT Research Repository.

The RMIT Research Repository is an open access database showcasing the research outputs of RMIT University researchers.

RMIT Research Repository: http://researchbank.rmit.edu.au/

\section{Citation:}

Van der Merwe, M, Minas, J, Ozlen, M and Hearne, J 2015, 'A mixed integer programming approach for asset protection during escaped wildfires', Canadian Journal of Forest Research, vol. 45, no. 4, pp. 444-451.

See this record in the RMIT Research Repository at:

https://researchbank.rmit.edu.au/view/rmit:31416

Version: Accepted Manuscript

Copyright Statement: ㄷ 2014, National Research Council of Canada.All rights reserved.

Link to Published Version:

http://dx.doi.org/10.1139/cjfr-2014-0239 


\title{
A mixed integer programming approach for asset protection during escaped wildfires
}

\author{
Martijn van der Merwe ${ }^{1}$, James P. Minas ${ }^{2}$, Melih Ozlen ${ }^{1}$, and John W. Hearne ${ }^{1}$ \\ ${ }^{1}$ School of Mathematical and Geospatial Sciences, RMIT University, GPO Box \\ 2476, Melbourne, Victoria, 3001, Australia \\ ${ }^{2}$ School of Business and Economics, Wilfrid Laurier University, Waterloo, Ontario, \\ Canada N2L 3C5.
}




\begin{abstract}
Incident Management Teams (IMTs) are responsible for managing the response to wildfires. One of the IMT's objectives is the protection of assets and infrastructure. In this paper we develop a mathematical model to assist IMTs in assigning resources to asset protection activities during wildfires.

We present a mixed integer programming model for resource allocation with the aim of protecting the maximum possible total value of assets. The model allows for mixed vehicle types with interchangeable capabilities, with travel times determined by vehicle specific speed and road network information. We define location specific protection requirements in terms of vehicle capabilities. The formulated model extends classic variants of the team orienteering problem with time windows.

The model capabilities are demonstrated using a hypothetical fire scenario impacting South Hobart, Tasmania, Australia. Computational testing shows that realistically sized problems can be solved within a reasonable time.
\end{abstract}

f Keywords: Forest fire; Emergency management; Integer programming; Team orienteering prob-

lem; Wildland fire 


\section{Introduction}

While fire is a natural component of many terrestrial ecosystems, uncontrolled wildfires occurring on populated landscapes can cause loss of life and damage to private property and community assets. In many jurisdictions including Australia, Canada and the United States, incident management teams (IMTs) are responsible for coordinating, planning and managing wildfire response related activities (Australasian Fire and Emergency Services Authorities Council 2013; ICS Canada 2012; US Department of Homeland Security 2008). IMTs dealing with large escaped wildfires operate in high pressure environments where they must make complex, time critical decisions. The tasks that the IMT has to perform include assessing the merits of the available information, devising strategies for containing the fire, minimising the impact of the fire, managing firefighting crews and other resources, issuing warnings to the public, and evacuating people. Factors affecting decisions include weather conditions, fire spread predictions, fuel state, assets under threat, the value of assets, and the location of vulnerable people. A strong need for decision support tools has been identified in the literature (McLennan et al. 2006; Omodei et al. 2005a,b). Challenges and difficulties faced include IMTs becoming overwhelmed with the volume of information, dealing with parameter uncertainty, and biases in human decision making. In this context, application of operations research and supply chain logistics tools such as assignment, routing and scheduling models could lead to enhanced management of large fires (Martell 2007).

The operations research literature concerning wildfire decision support has been reviewed by Martell (1982), Martell et al. (1998) and most recently by Minas et al. (2012). Models developed to support short term IMT level wildfire decision making have been largely concerned with the dispatch of resources to fires and with fire-line construction. Mees et al. (1994) considered the problem of optimally assigning resources to fireline construction in order to minimise the total expected cost plus loss. Their model calculates the probability of fire containment as a function of resource allocation, taking into account uncertainty in both the flame length of the fire and in the width of fireline that is produced. Martin-Fernández et al. (2002) developed a model that employs discrete simulation algorithms and Bayesian optimisation methods for real-time dispatch of firefighting resources to wildfires. The model was applied to a case study in Northern Spain to demonstrate its ability to handle real-time weather changes and chaotic fire behaviour. An integer programming model to determine the optimal mix of firefighting resources to dispatch to a given fire to achieve containment with minimal resultant costs and damages was described by Donovan and Rideout (2003). $\mathrm{Hu}$ and Ntaimo (2009) presented a stochastic mixed-integer programming model for initial attack. Pappis and Rachaniotis (2010a) considered the non-linear dynamics of fire suppression, that is the observation that small delays in dispatch of initial attack resources can result in dramatic fire-loss increases. They constructed a non-linear programming model which utilises the concept of 'deteriorating jobs', that is, jobs processed later in the sequence require more time. Pappis and Rachaniotis (2010a) scheduled a single firefighting resource when there are several existing fires to be controlled. The model was later extended to allow scheduling of multiple firefighting resources (Pappis and Rachaniotis 2010b). Ntaimo et al. (2012) presented a two-stage stochastic integer programming model for initial attack with the goal of containing as many fires as possible while minimising rental and travel cost and the expected future maintenance cost. The model allows for multiple types of firefighting resources and defines a standard response based on fireline production rates. A statistical sampling approach is employed to handle the large number of possible scenarios of fire occurrence and fire behaviour. Haight and Fried (2007) presented a scenario-optimisation integer programming model for initial attack resource deployment based on 
the classical maximal covering location model. The model's objective is to minimise the expected number of fires that do not receive a standard response subject to resource availability constraints, where a standard response is defined as the required number of resources that can reach the fire within a maximum response time. The optimisation model was later expanded by Lee et al. (2013), allowing the sharing of resources between multiple planning units and adding different resource types that differ from one another with respect to response time, fireline production, cost, and basing constraints. Homchaudhuri et al. (2013) presented a genetic algorithm based approach to efficiently allocate resources to fireline construction and optimum fireline building that minimises the total damage due to wildfires.

On days of extreme fire weather, when large fires are burning in hot, dry and windy conditions, fire suppression may be both ineffective and unsafe. In these circumstances, fire agency resources may be better utilised by assigning them to "defensive" tasks such as: asset protection, protecting vulnerable people in place, evacuating communities, collecting information and issuing warnings (CSIRO 2009).

In this paper, we consider the problem of assigning resources to asset protection activities when large wildfires are burning out of control and fire suppression is not a viable option. We formulate a mixed integer programming model assigning resources to asset protection with the aim of maximising the total saved asset value. The model allows for mixed vehicle types with interchangeable capabilities with vehicle travel times determined by vehicle specific speeds and road network information. The protection requirements of locations are defined in terms of the vehicles' capabilities. To the best of our knowledge, this is the first time that the pre-emptive protection of community assets and infrastructure from advancing wildfire has been described and modelled.

The remainder of the paper is structured as follows. A description of the wildfire asset protection problem is provided in the next section. Similarities of the wildfire asset protection problem to the team orienteering problem are discussed. A mixed integer programming formulation of the problem is presented and explained in $\S 3$. This is followed by a discussion in $\S 4$ of the model parameters and how different conditions and scenarios could be parameterised. The models functionality is then demonstrated on a hypothetical wildfire scenario in South Hobart, Tasmania, Australia. The model's computational performance is evaluated in $\S 5$. The paper concludes with a discussion of the results and possible future research directions.

\section{The wildfire asset protection problem}

When escaped wildfires impact communities and infrastructure, it is often possible to carry out a number of activities to protect the assets being threatened. Wetting down structures, clearing gutters of combustible material and putting out spot fires are a few examples. The responding fire services need to decide how best to assign the available resources to these asset protection tasks at various locations.

To aid the IMT in their efforts, in some Australian jurisdictions the fire services prepare community protection plans (D. Killalea, Tasmania Fire Service, personal communication, 2014). These plans, among other things, identify various community assets together with information pertinent to protecting those assets. The protection plans contain GPS coordinates, access information, number and type of resources required to protect the assets and importance of the assets to the community. Some examples of community assets are communication towers, hotels, historically significant buildings, schools, bridges, factories and hospitals. 


\subsection{Asset value and protection requirements}

Each asset under threat is assigned a protection priority by the IMT either explicitly or implicitly. For modelling purposes, this protection priority is translated into a value, and the aim is to protect the maximum total value of assets with the available limited resources. The value of locations may be expressed as a monetary amount or a relative value. Often values would be perceived values rather than calculated values. Communities are often consulted to aid in assessing the value of an asset. A factor that may be considered is the contributions of an asset to the recovery of a community after a wildfire, for example, communication infrastructure is key in coordinating relief efforts. Values can be determined using existing operations research techniques such as analytic hierarchy process.

The community protection plans identify the protection requirements for each asset. For an asset to be protected, the resources with the required capabilities must arrive in a timely manner and remain at the asset for a sufficient period of time, called the service duration, to carry out the necessary protection tasks. The number and type of resources required to provide the adequate level of protection to an asset will depend on a number of factors. Examples are the accessibility of the location, whether four wheel drive vehicles are required, the availability of reticulated water, and the type of protection activities required to protect the asset.

\section{$2.2 \quad$ Resources}

The typical resource units being assigned are fire trucks, commonly referred to as tankers. Besides tankers, various types of vehicles may be available to the IMT to utilise in dealing with a fire threatening a community. As an example, the various vehicles and resources of the Tasmania Fire Service are shown in Table 1. The average travelling speeds of each vehicle type are summarised in Table 2.

\subsection{Time windows}

The advancing fire fronts impose time constraints on protection activities. These time constraints can be translated into time windows during which asset protection tasks must commence in order to be successful. The time that a resource starts working on a task is called the service start time. The time windows are determined by the anticipated time to impact, this is the time remaining before the asset is impacted by the fire. The time to impact may be estimated using fire spread modelling. Extensive research has been carried out in the modelling and prediction of fire spread, which is summarised in a series of reviews undertaken by Sullivan (2009a,b,c).

\subsection{Related problems}

The problem of assigning tasks to resources during large escaped wildfires as described above has features in common with the team orienteering problem with time windows (TOPTW). In the TOPTW, a team of orienteerers have a limited time to collect rewards from various locations. The reward at each location is only available for a period of time specified by the location's time window.

The TOPTW is in the class of vehicle routing problems with profits and is closely related to the selective vehicle routing problem with time windows, which generalises the TOPTW by adding a capacity constraint to each vehicle (Feillet et al. 2005). Vehicle routing problems with profits have been reviewed by Archetti et al. (2013) and orienteering problems by Vansteenwegen et al. (2011). 
Table 1: Vehicle types, their abbreviation, typical crew capacity and roles that the vehicle can perform.

\begin{tabular}{|c|c|c|c|}
\hline Vehicle type & Abbreviation & $\begin{array}{l}\text { Typical } \\
\text { crew size }\end{array}$ & Capabilities and limits \\
\hline Heavy pumper & $\mathrm{HP}$ & 4 & $\begin{array}{l}\text { Asset protection; limited to formed roads, both } \\
\text { sealed and ununsealed; limited to reticulated water }\end{array}$ \\
\hline Medium pumper & MP & 4 & $\begin{array}{l}\text { Asset protection; limited to formed roads, both } \\
\text { sealed and unsealed; limited to reticulated water }\end{array}$ \\
\hline Heavy tanker & $\mathrm{HT}$ & 4 & $\begin{array}{l}\text { Suppression and asset protection; roads } \\
\text { (formed/unformed and } 4 \mathrm{WD} \text { vehicular tracks) }\end{array}$ \\
\hline Medium tanker & MT & 4 & $\begin{array}{l}\text { Suppression and asset protection; roads } \\
\text { (formed/unformed and } 4 \mathrm{WD} \text { vehicular tracks) }\end{array}$ \\
\hline Light tanker & LT & 2 & $\begin{array}{l}\text { Suppression and asset protection; roads } \\
\text { (formed/unformed and } 4 \mathrm{WD} \text { vehicular tracks) }\end{array}$ \\
\hline Hydraulic ladder & Aer & 2 & $\begin{array}{l}\text { Asset protection; limited to formed roads (sealed, } \\
\text { unsealed) and reticulated water (e.g. Snorkel) }\end{array}$ \\
\hline Transportation vehicle & Trans & $<10$ & $\begin{array}{l}\text { Information gathering, firefighter transport (e.g. } \\
\text { troop carrier) }\end{array}$ \\
\hline Miscellaneous vehicle & Misc & - & $\begin{array}{l}\text { Miscellaneous; limited to formed } \operatorname{roads}(e . g . \text { canteen } \\
\text { vehicle) }\end{array}$ \\
\hline Dozer & - & - & Fire break construction \\
\hline Excavator & - & - & Fire break construction \\
\hline
\end{tabular}

Drawing the analogy to the TOPTW, fire tankers may be seen as members of an orienteering team. The assets requiring protection are equivalent to control points, each with an associated time window and value. However, in the wildfire asset protection problem, multiple resources are often required to protect a single asset, whereas the TOPTW requires the visit of only a single team member to claim a reward from a location. In Van der Merwe et al. (2014) a new model, the cooperative orienteering problem with time windows (COPTW), was formulated to address this shortcoming.

The COPTW generalises the TOPTW to allow multiple resources to converge on a single location and cooperatively collect the associated reward. In this paper, we further extend the cooperative orienteering problem to allow for mixed resource types with different interchangeable capabilities, asset protection requirements defined in terms of those capabilities, and vehicle specific speed and the condition of the road network determining the travelling time of each vehicle.

\section{Model formulation}

The resource units will be referred to as vehicles. Let $\mathcal{Q}$ be the set of vehicle types. There is a total of $p_{q}$ vehicles of each type $q \in \mathcal{Q}$ available for assignment. The value of the asset at location $i$ is $v_{i}$. Let $a_{i}$ be the service duration associated with location $i$, that is the duration vehicles are required stay at the location to protect the asset. Each asset has an associated time window specifying the time during which protection activities must commence in order to be successful. The earliest time 
Table 2: The average travelling speeds of the different vehicle types.

\begin{tabular}{llccccc}
\hline Transport class & Surface type & Default & HP \& MP & HT & MT & LT \\
\hline National/State Highway & Sealed & 100 & 90 & 80 & 80 & 90 \\
Major arterial road & Sealed & 80 & 75 & 75 & 75 & 75 \\
Major arterial road & Unsealed & 80 & 60 & 60 & 60 & 60 \\
Arterial road & Sealed & 80 & 70 & 70 & 70 & 70 \\
Arterial road & Unsealed & 60 & 60 & 60 & 60 & 60 \\
Feeder & Sealed & 80 & 60 & 50 & 60 & 60 \\
Feeder & Unsealed & 60 & 50 & 50 & 50 & 50 \\
Access road & Sealed & 60 & 40 & 40 & 40 & 40 \\
Access road & 4WD / Unsealed & 20 & - & 20 & 20 & 20 \\
Vehicular track & 4WD / Unsealed & 20 & - & 10 & 10 & 10 \\
\hline
\end{tabular}

that protection activities may commence is $o_{i}$, also called the time window's opening time. The latest time that protection activities may commence is $c_{i}$, called the time window's closing time. We assume that service has to be provided cooperatively by the required resources, and that the sequence in which service is delivered at each asset is not important.

\subsection{Depots}

Initially the vehicles are located at one of $m$ depots at locations $1, \ldots, m$. The depot may be a vehicle storage area, a fire station or a staging area. For brevity these locations will be referred to as depots. There are $s_{t o c k}$ vehicles of type $q$ stationed at depot $i$. The assets are located at locations $m+1, \ldots, n-1$. Note that location $n$ is a dummy location representing the sink in the model formulation. Further $a_{i}=0$ for all $i=1, \ldots, m$. The departure of vehicles from a depot may be delayed by specifying $o_{i}>0$.

\subsection{Asset protection requirements}

Let $\mathcal{U}$ be the set of vehicle capabilities. Each vehicle type $q$ has an associated capability vector $\boldsymbol{c a p}_{q}$. The protection requirement for each location is defined in terms of the capabilities required to protect the assets at that location.

The protection requirement of an asset $i$ is given by the protection vector $\boldsymbol{r}_{i}$ specifying the amount of each capability required. An asset is considered protected if the combined capabilities of the vehicles assigned to the asset meets or exceeds the capabilities required. Furthermore, the vehicles must arrive before or at the start of service time $S_{i}$ and stay for the service duration $a_{i}$.

For example, one way of satisfying the protection vector $\boldsymbol{r}_{i}=(2,3)$ is by combining the following three vehicles; one vehicle with $\boldsymbol{c} \boldsymbol{a} \boldsymbol{p}_{1}=(2,1)$ and two vehicles with $\boldsymbol{c a} \boldsymbol{p}_{2}=(0,1)$.

\subsection{Travel time}

The time it takes for a vehicle to travel between two locations will depend on the vehicle type and the roads being used. Further, certain roads may only be accessible by some vehicle types, for example roads accessible only by four wheel drive vehicles. As a result, each vehicle type will often 
have a unique travel time between two locations. The travel time from location $i$ to location $j$ is denoted by $t_{i j q}$ for each vehicle type $q$.

\subsection{Preprocessing}

We eliminate the paths that are not feasible due to the time window constraints. This preprocessing approach is equivalent to that of Van der Merwe et al. (2014). Let $\mathcal{L}$ be the set of all possible location pairs. For vehicles of type $q$, consider two locations $i$ and $j$ chosen such that the earliest possible departure from location $i$ results in an arrival at location $j$ which is later than the closing time of location $j$. Since no feasible solution will require vehicles of type $q$ to travel from $i$ to $j$, it is possible to ignore this route. Let $\mathcal{E}_{q}$ be the index set including only feasible routes, that is $(i, j) \in \mathcal{E}_{q}$ if and only if $(i, j) \in \mathcal{L}$ and $o_{i}+a_{i}+t_{i j q} \leq c_{j}$.

Two sets $\mathcal{F}_{q}^{k}$ and $\mathcal{G}_{q}^{k}$ are defined to simplify the model notation: $\mathcal{F}_{q}^{k}$ is the index set of locations adjacent to location $k$, that is $i \in \mathcal{F}_{q}^{k}$ if $(i, k) \in \mathcal{E}_{q}$, and $\mathcal{G}_{q}^{k}$ is the index set of locations adjacent from location $k$, that is $j \in \mathcal{G}_{q}^{k}$ if $(k, j) \in \mathcal{E}_{q}$.

\subsection{The mixed integer programming model formulation}

The following decision variables are used in the model formulation:

- $X_{i j q}$ is an integer decision variable indicating the number of vehicles of type $q$ travelling from location $i$ to location $j$;

- $Y_{i}=1$ if asset $i$ is protected, otherwise $Y_{i}=0$;

- $Z_{i j q}=1$ if a vehicle of type $q$ is travelling from location $i$ to location $j$, otherwise $Z_{i j q}=0$; and

- $S_{i}$ for all $i \in 1, \ldots, n$ is the start time of service at location $i$.

Based on the notation introduced above, the problem being considered may be formulated as a mixed integer programming problem:

$$
\begin{gathered}
\text { Maximise } \sum_{i=m+1}^{n-1} v_{i} Y_{i} \\
\text { subject to } \\
\sum_{j \in \mathcal{G}_{q}^{k}} X_{k j q}=\text { stock }_{k q} \quad \forall k=1, \ldots, m, q \in \mathcal{Q} ; \\
\sum_{i \in \mathcal{F}_{q}^{k}} X_{i k q}=\sum_{j \in \mathcal{G}_{q}^{k}} X_{k j q} \quad \forall k=m+1, \ldots, n-1, q \in \mathcal{Q} ; \\
\sum_{q \in \mathcal{Q}} \sum_{i \in \mathcal{F}_{k}^{q}} X_{i k q} c a p_{q u} \geq r_{k u} Y_{k} \quad \forall u \in \mathcal{U}, k=m+1, \ldots, n-1 ; \\
X_{i j q} \leq p_{q} Z_{i j q} \quad \forall(i, j) \in \mathcal{E}_{q}, q \in \mathcal{Q} ; \\
S_{i}+t_{i j q}+a_{i}-S_{j} \leq M\left(1-Z_{i j q}\right) \quad \forall(i, j) \in \mathcal{E}_{q}, q \in \mathcal{Q} ; \\
o_{i} \leq S_{i} \quad \forall i=1, \ldots, n-1 ;
\end{gathered}
$$




$$
\begin{gathered}
S_{i} \leq c_{i} \quad \forall i=1, \ldots, n-1 ; \\
X_{i j q} \in\left\{0,1,2, \ldots, p_{q}\right\}, Z_{i j q} \in\{0,1\} \quad \forall(i, j) \in \mathcal{E}_{q}, q \in \mathcal{Q} ; \\
Y_{i} \in\{0,1\} \quad \forall i=m+1, \ldots, n-1 .
\end{gathered}
$$

The objective function (1) maximises the total protected asset value. Constraints (2) define the starting position of vehicles as depots. The vehicle flow to and from each location is balanced by constraints (3). Constraints (4) enforce the condition that an asset is protected only if the vehicles assigned to the asset collectively meet the protection requirement. Constraints (5) and (6) ensure that service at a location may only start after protection activity at a previously visited location has been completed and sufficient time for travel has been allowed, with $M$ representing a large constant. Setting $M=\max \left(o_{i}\right)+\max \left(t_{i j q}\right)+\max \left(a_{i}\right)-\min \left(c_{i}\right)$ is sufficiently large for this purpose. The start of protection activities at locations are limited to their respective time windows by constraints (7) and (8). Constraints (9) and (10) enforce the integer and binary conditions on the appropriate decision variables.

\section{Model demonstration}

In this section we demonstrate how the model could be used in practice. The modelling approach's flexibilities are discussed with regards to protection activities and interchanging or combining resources to protect assets. Finally a case study is considered using assets located in South Hobart, Tasmania, Australia.

\subsection{Time windows}

There are two types of tasks considered in this study; active defence tasks and strategic defence tasks. Active defence tasks are those tasks that take place during the time that a fire is actively impacting the assets, either through direct flame contact or embers. Examples of active defence tasks are putting out spot fires near assets and wetting down structures. The duration of active tasks depend on the intensity of the fire, the structure being threatened and the fuel surrounding the asset, but typically is between fifteen minutes and six hours. To ensure active protection activities commence at the time of impact, the time window's opening time is equal to its closing time, i.e. time windows represent a single point in time.

Strategic defence tasks are preparatory tasks that can be carried out before a fire impacts an asset. Examples of strategic defence tasks include: clearing fuel around a structure, wetting down the roof, setting up a sprinkler system and applying fire retardant expansion foam to a structure. The time windows associated with strategic defence tasks start some time before the anticipated time of impact and close near the time of impact, depending on the activity.

\subsection{Interchanging resources}

The model allows for combining and substituting resources to meet a given location's protection requirements. Although a myriad of possibilities of interchanging and combining resources to meet protection requirements exist, three cases are discussed next as an illustration.

Possibly the simplest case is when there is no overlap in the capability of vehicle types. Consider the following example, two vehicles with capabilities $\boldsymbol{c} \boldsymbol{a} \boldsymbol{p}_{1}=(1,0)$ and $\boldsymbol{c a p}_{2}=(0,1)$, respectively, are not substitutable. 
The second case is where vehicles can perform the same task, but some vehicles can provide more of a required capability than others. In this case the vehicle capability vectors are a scalar multiple of each other. As an example, assigning a vehicle with $\boldsymbol{c a p}_{3}=(2,4)$ to protect an asset, is the same as assigning two vehicles with $\boldsymbol{c a p}_{4}=(1,2)$.

The third case is where one vehicle can perform the role of another, but not vice versa. For example, tankers can replace pumpers, but since pumpers do not carry their own water supply, they cannot always replace tankers. Pumpers can only operate where a water source is available, tankers on the other hand, do have their own water supply and are not limited by the availability of water. To illustrate how this would be handled in the model, consider the vehicle capabilities contained in Table 3.

Table 3: The capability vectors for each vehicle type to demonstrate how resource substitution may occur.

\begin{tabular}{lc}
\hline Vehicle type $(q)$ & $\boldsymbol{c a p}_{q}$ \\
\hline Light tanker $(\mathrm{LT})$ & $(1,1)$ \\
Heavy tanker (HT) & $(2,2)$ \\
Medium pumper $(\mathrm{MP})$ & $(0,4 / 3)$ \\
Heavy pumper $(\mathrm{HP})$ & $(0,2)$ \\
\hline
\end{tabular}

Consider an asset $i$, which has no water source, that has a protection requirement of $\boldsymbol{r}_{i}=(2,2)$. The first entry indicates that the location requires tankers and the second entry indicates that a heavy vehicle (or equivalent) is required. The protection requirement of asset $i$ can be met by either one heavy tanker, since $\boldsymbol{c} \boldsymbol{a} \boldsymbol{p}_{H T}=(2,2) \geq(2,2)=\boldsymbol{r}_{i}$, or two light tankers, since $2 \cdot \boldsymbol{c} \boldsymbol{a} \boldsymbol{p}_{L T}=$ $2 \cdot(1,1) \geq(2,2)=\boldsymbol{r}_{i}$. Note that no combinations of pumpers can satisfy the protection requirement.

Next consider a location at location $j$ that has a reticulated water source and a protection requirement of $\boldsymbol{r}_{j}=(0,2.5)$. The protection requirement of location $j$ may be met by two medium pumpers, since $2 \cdot \boldsymbol{c a} \boldsymbol{p}_{M P}=2 \cdot(0,4 / 3) \geq(0,2.5)=\boldsymbol{r}_{j}$, a medium pumper and a heavy pumper $\boldsymbol{c a p}_{M P}+\boldsymbol{c a p}_{H P}=(0,4 / 3)+(0,2) \geq(0,2.5)=\boldsymbol{r}_{j}$, or two heavy pumpers, $2 \cdot \boldsymbol{c a} \boldsymbol{p}_{H P}=2 \cdot(0,2) \geq$ $(0,2.5)=\boldsymbol{r}_{j}$. The protection requirement can also be met by the appropriate combination of tankers. For example, a heavy tanker and a light tanker would meet the protection requirement, since $\boldsymbol{c a p}_{H T}+\boldsymbol{c} \boldsymbol{a} \boldsymbol{p}_{L T}(1,1)+(2,2) \geq(0,2.5)=\boldsymbol{r}_{i}$.

The entries in the capability vectors may be viewed as resources being delivered to a location by the vehicle. For example, the vehicle capacity vector could specify the number of people and litres of water each vehicle can deliver per minute. Each location's protection requirement may specify how much of each resource (i.e. people and water) is required to protect the assets at that location.

\subsection{Case study: South Hobart}

In January of 2013, several fires burned out of control near Hobart with devastating consequences. Among the losses were 203 residential buildings, approximately $662 \mathrm{kms}$ of commercial fencing and 10000 head of livestock, mainly sheep. The estimated cost of the losses was in the order of AUD100 million, not taking into account the cost of emergency response and recovery operations and the longer term economic impact (Hyde 2013).

Fire stations located in Hobart and assets specified in the South Hobart protection plan are used in the demonstration. The location of these fire stations and assets are shown in Figure 1 
which also contains the parameter values for these locations.

In our scenario a simple fire spread, radiating outwards at rate of $3 \mathrm{~km} / \mathrm{h}$ from a single point of origin in a circular fashion impacting South Hobart, is assumed. Each asset requires 30 minutes of active defence commencing at the time of impact. The travel times between assets were calculated using Google Maps' Distance Matrix service. We assume that there are four vehicle types. The capability of each vehicle is shown in Table 3. The first entry indicates whether the vehicle requires an reticulated water source. The second entry indicates the vehicles size, or litres of water per minute it can provide. A total of 14 vehicles are available for assignment: 5 light tankers, 2 heavy tankers, 5 medium pumpers and 2 heavy pumpers. Each asset is randomly assigned a value of either 10,20 or 30 , each with equal probability.

The scenario is solved for two variations considering different starting locations of vehicles. In the first case, all vehicles are located at a fire station on the eastern side of the Derwent river. The optimal assignment of vehicles is shown in Figures 2 and 3. In the second variation, vehicles are distributed among the various fire stations. An optimal solution is shown in Figures 4 and 5.

In the second variation considered, a total value of 270 is protected, compared to 240 in the first variation. The reduced protected value is due to the increased travel distances. For example, in the first variation the required resources can't reach the three eastern assets 11, 12 and 14 in time. In the second variations resources are located closer to these assets and asset 12 is saved. High value assets are prioritised over low value assets, for example the southern assets 1 and 2 are unprotected in both cases. In this scenario, tankers, not being reliant on a water source, are the highest utilised resource with all the tankers assigned to protection activities while some pumpers remained unassigned. It is also interesting to note that some roads are heavily utilised, giving an indication of which roads are critical to keep open.

Optimal solution for both of these problem instances could be found within 2 seconds using CPLEX 12.6 on a desktop computer. The computational behaviour of the model is explored in the next section.

\section{Computational study}

Computational testing was carried out on a single node of a computer cluster. The node has two Intel Xeon E5-2670 processors and 64GB of RAM. CPLEX 12.6 was used to solve the problem instances and performance was measured in elapsed time (wall-clock time). The solver's parallel optimisation mode was set to deterministic while all the remaining CPLEX solver parameters were left at their default values.

Ten problem instances with 60 locations each were generated. The location of assets are randomly chosen with a uniform probability density function inside a $80 \mathrm{~km}$ by $80 \mathrm{~km}$ square. The travel times between locations were calculated by taking the direct distance between the locations and using a travel speed of $60 \mathrm{~km} / \mathrm{h}$.

The opening time of each time window is correlated to the $x$-coordinate of it's location. The opening time is given by $o_{i}=10 x_{i}$, which translate to a fire spreading across the landscape at a rate of $10 \mathrm{~km} / \mathrm{h}$. It is assumed that all the time windows have the same length $w$, the closing time of each window is thus given by $c_{i}=o_{i}+w$. Location values are taken from the well known orienteering problem benchmark instance r101 (Christofides et al. 1979), while each entry of the protection requirement vectors were randomly selected, with equal probability, from the set $\{1,2,3\}$. The smaller problem instances (30,40 and 50 locations) are subsets of the 60-location instances. 
Figure 1: Assets located in South Hobart and Hobart fire stations (Map data (C)2014 Google).

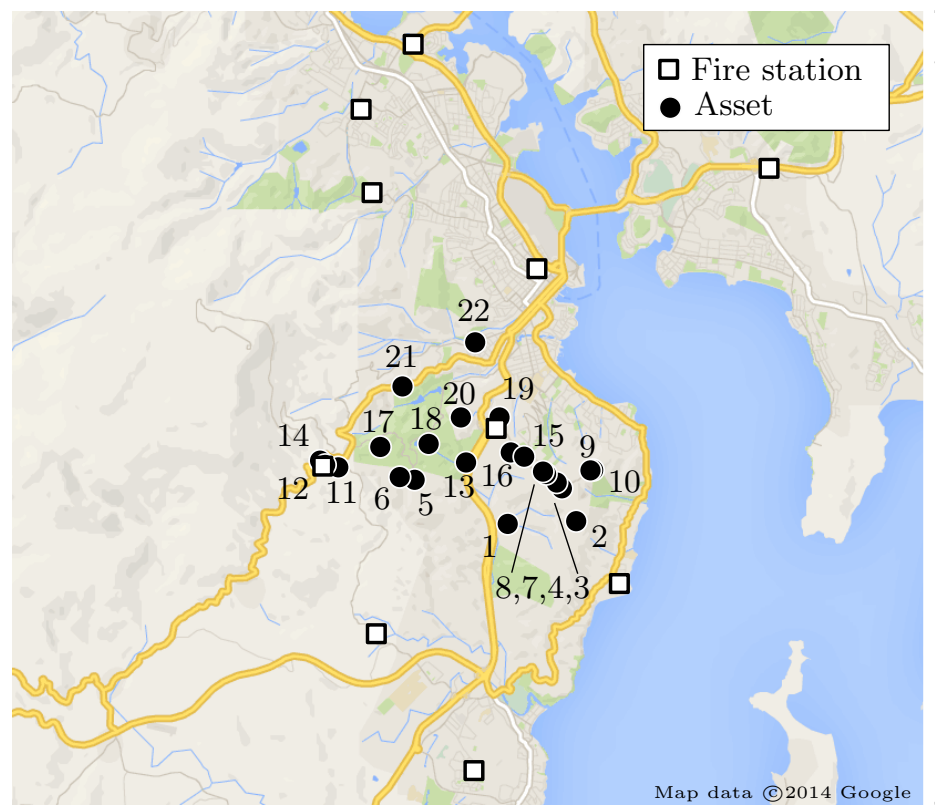

\begin{tabular}{cccc}
\hline$i$ & $v_{i}$ & $o_{i}=c_{i}$ & $\boldsymbol{r}_{i}$ \\
\hline 1 & 10 & 91 & $(2,2)$ \\
2 & 10 & 126 & $(3,2)$ \\
3 & 30 & 119 & $(1,1)$ \\
4 & 30 & 117 & $(2,3)$ \\
5 & 20 & 45 & $(3,1)$ \\
6 & 10 & 38 & $(3,1)$ \\
7 & 30 & 112 & $(2,3)$ \\
8 & 10 & 110 & $(3,2)$ \\
9 & 20 & 134 & $(2,3)$ \\
10 & 10 & 136 & $(1,1)$ \\
11 & 30 & 8 & $(1,3)$ \\
12 & 30 & 2 & $(1,1)$ \\
13 & 10 & 72 & $(3,3)$ \\
14 & 10 & 0 & $(2,2)$ \\
15 & 10 & 102 & $(2,3)$ \\
16 & 10 & 95 & $(1,2)$ \\
17 & 20 & 31 & $(1,2)$ \\
18 & 20 & 55 & $(2,3)$ \\
19 & 10 & 93 & $(2,1)$ \\
20 & 20 & 75 & $(1,1)$ \\
21 & 20 & 51 & $(3,1)$ \\
22 & 30 & 94 & $(2,2)$ \\
\hline
\end{tabular}

Figure 2: An optimal solution for the first variation described in the text. All of the vehicles are located at a fire station on the eastern side of the Derwent river (Map data (C)2014 Google).

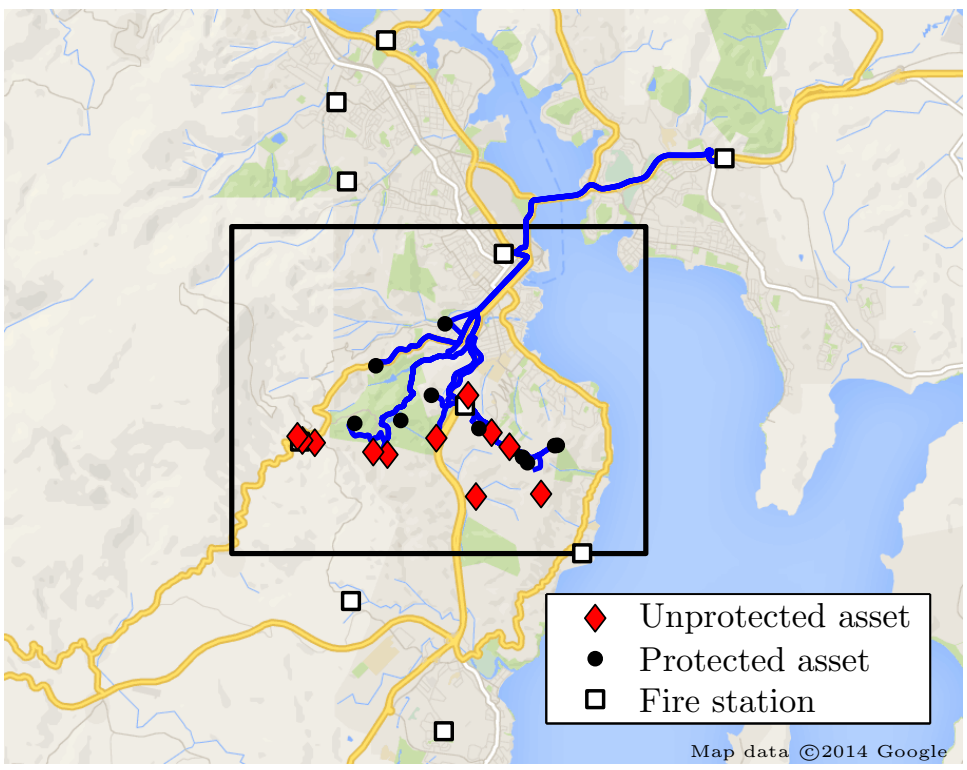


Figure 3: The solution in Figure 2 shown by vehicle type. The map has been cropped to the area highlighted by the rectangle in Figure 2 (Map data (C)2014 Google).

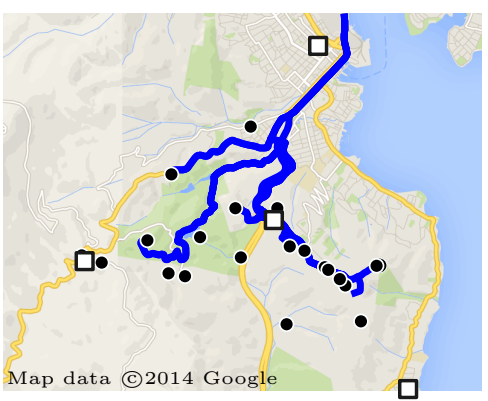

(a) Light tankers

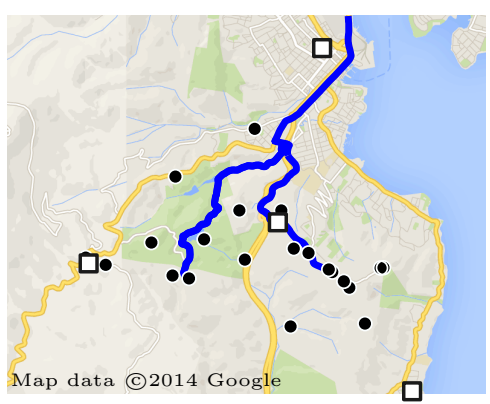

(c) Medium pumpers

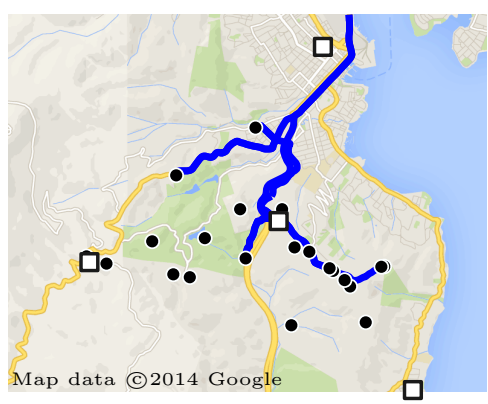

(b) Heavy tankers

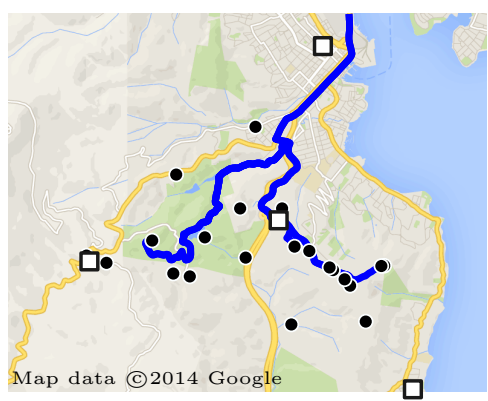

(d) Heavy pumpers

Figure 4: An optimal solution for the second variation described in the text. The vehicles are located at various fire stations across Hobart (Map data (C)2014 Google).

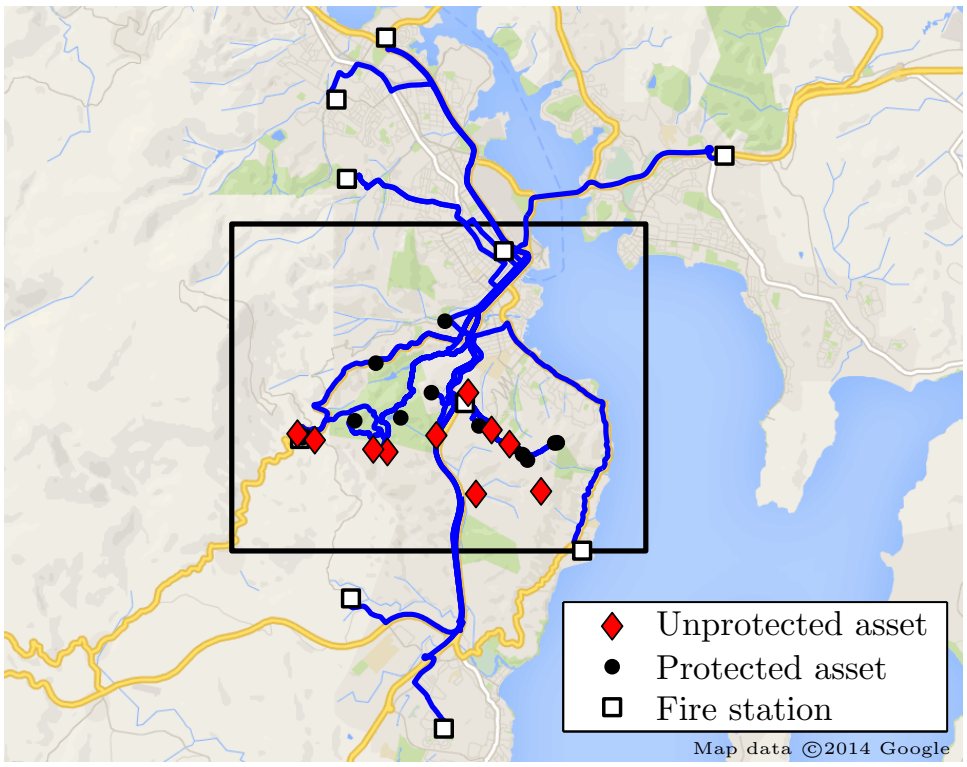


Figure 5: The solution presented in Figure 4 shown by vehicle type. The map has been cropped to the area highlighted by the rectangle in Figure 4 (Map data (C)2014 Google).

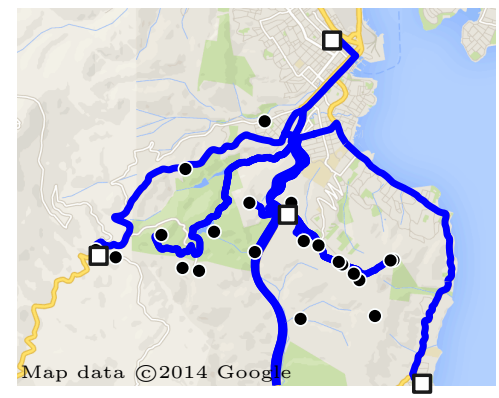

(a) Light tankers

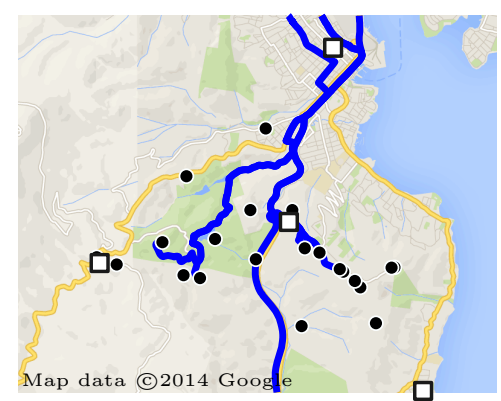

(c) Medium pumpers

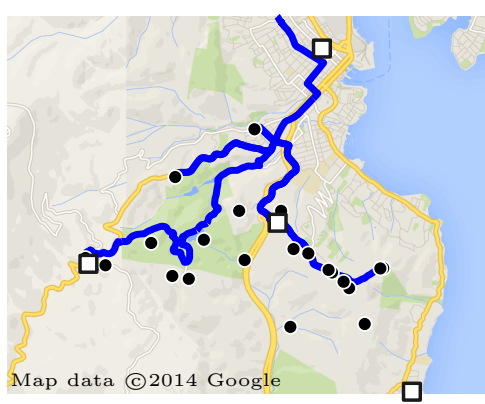

(b) Heavy tankers

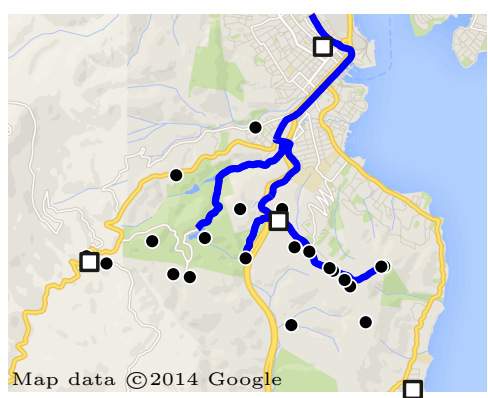

(d) Heavy pumpers 
Our first set of experiments considers only two entries in the vehicle capability vectors and four vehicle types. The rest of the parameters are set as summarised in Table 4 . The results of these experiments are contained in Table 5(a). The problems generally become harder as the number of locations and vehicles increase. Problems of size 30 are generally quick to solve, while the solution time for larger problems typically depends on their properties. Larger time windows result in harder problems.

Table 4: The parameter values used for computational testing.

\begin{tabular}{lcccc}
\hline Parameter & \multicolumn{4}{c}{ Value } \\
\cline { 2 - 5 } & $q=1$ & $q=2$ & $q=3$ & $q=4$ \\
\hline $\boldsymbol{c a p}_{q}(|\mathcal{U}|=2)$ & $(1,1)$ & $(2,1)$ & $(0,2)$ & $(1,0)$ \\
$\boldsymbol{c a p}_{q}(|\mathcal{U}|=3)$ & $(1,1,2)$ & $(2,1,0)$ & $(0,2,1)$ & $(1,0,1)$ \\
$p_{q}(p=6)$ & 2 & 1 & 2 & 1 \\
$p_{q}(p=10)$ & 3 & 2 & 3 & 2 \\
\hline
\end{tabular}

The second set of experiments consider three entries in the vehicle capability vectors. The rest of the parameters are set as summarised in Table 4. The results of these experiments are available in Table 5(b). Although all the problems of size 30 considered could be solved within a couple of minutes, increasing the number of elements in the vehicle capability vector increased the solution times.

Table 5: The solution times for test instances in seconds. The number of unsolved problems after twenty minutes elapsed time (wall time) is indicated in parenthesis while a dash indicates that none of the problems could be solved within the twenty minute time limit.

(a) $|\mathcal{U}|=2$

\begin{tabular}{rrrrr}
\hline & $p \rightarrow 6$ & 10 & 6 & 10 \\
$n$ & $w \rightarrow 20$ & 20 & 40 & 40 \\
\hline 30 & 2 & 1 & 42 & 37 \\
40 & 6 & 7 & $(1) 178$ & $(6) 939$ \\
50 & 12 & 48 & $(5) 819$ & - \\
60 & 31 & $(2) 422$ & - & - \\
\hline
\end{tabular}

(b) $|\mathcal{U}|=3$

\begin{tabular}{rrrrr}
\hline & $p \rightarrow 6$ & 10 & 6 & 10 \\
$n$ & $w \rightarrow 20$ & 20 & 40 & 40 \\
\hline 30 & 3 & 2 & 34 & 113 \\
40 & 9 & 18 & $(1) 209$ & $(7) 1060$ \\
50 & 21 & 99 & - & - \\
60 & 34 & $(4) 673$ & - & - \\
\hline
\end{tabular}

These results indicate that problems containing 50 locations or more are very hard to solve with this integer programming approach. 


\section{Discussion}

In this paper we presented a mixed integer programming approach to the problem of protecting assets during large escaped wildfires. This research is a step towards providing IMTs with tools that may be used in real-time to reduce the impact of wildfires on communities.

The mixed integer programming model presented here generalises the COPTW by allowing mixed vehicle types, introducing a vector specifying the protection requirement for each location and allowing each vehicle type to have a unique travel time between two locations.

The working of the model was demonstrated using the locations of assets and fire stations in Hobart, Tasmania, Australia. Although parts of the data used to demonstrate the model was sourced from Tasmania Fire Service, the modelling approach is general and the model could be applied to other locations.

Testing of the asset protection model formulation demonstrated that it is computationally feasible to apply the model to real life asset protection problems. However, as the problem size increases the model becomes harder to solve. We also showed that the solution time depends on the properties of the problem. With this in mind, the development of methods to improve solution times could prove beneficial. Potential approaches may employ CPLEX as a heuristic solver to find a good enough solution or the development of meta-heuristic solution techniques. The question then arises which heuristics methods are most suitable and when may a solution be considered of acceptable quality? Heuristic techniques have already proved very useful for solving larger instances of the team orienteering problem with time windows within seconds. Techniques to solve the team orienteering problem often rely on the ability to independently generate paths for each vehicle, calculating the contribution of each individual vehicle to the overall objective function. The cooperative element of our approach does not allow the independent calculation of the contribution of a vehicle to the objective function, which makes it impossible to apply these techniques directly to the wildfire asset protection problem. Therefore, further research is required to assess the effectiveness of heuristics in solving the problem described here.

Future work may consider modifications to the model presented here to account for certain wildfire management cases. For instance, saving lives is always the top priority. In the case where lives are at risk, the evacuation and protection of vulnerable people in place would need to be carried out before any asset protection is considered. This may be accounted for in the model by the addition of constraints. Other extensions may require more in-depth reformulation of the model. As an example, when it is not possible to protect an asset completely, it may still be possible to partially save or reduce the damage to an asset. In the model this would translate to collecting partial rewards from locations.

The parameter values are not always known with certainty. Uncertainty could be accounted for by conservatively assigning vehicles to asset protection tasks, for example requiring vehicles to arrive earlier and depart later from the protected location. This would be done by increasing the service time and having the time window's closing time earlier. It is also possible to resolve the model once new information emerges, similar to the approach in dynamic vehicle routing problems. In some cases there may be some expectation associated with the problem's parameter values, for example predicted wind changes may determine a probability distribution of time windows and probabilistic fire spread models could provide a likelihood of an asset being impacted. Theoretically stochastic programming may deal with these cases, but in practice, this approach might prove computationally intractable and heuristics might need to be explored.

In our model we assumed that vehicles will be engaged continuously at a location for the entire 
service duration to carry out the required protection activities. The service at a location can be split into separate tasks, with vehicles only required to be present at an asset for the appropriate tasks. Having one or more tasks at a location can be modelled by representing each asset with multiple nodes, a node for each protection task to be carried out. To collect the reward (or save the asset) service must be delivered to all the nodes belonging to a single asset. Each node having an opening time, a closing time and a service duration. The travel time between nodes belonging to the same location would be zero. This approach may also be used to model the case where either an active or a strategic defence task can be carried out to protect an asset.

\section{Acknowledgements}

We would like to thank Damien Killalea from the Tasmania Fire Service for providing valuable information on wildfire incident management. This work was supported by the Bushfire CRC in the form of scholarship funding to Martijn van der Merwe. The third author is supported by the Australian Research Council under the Discovery Projects funding scheme (project DP140104246). Computational testing was carried out on Trifid, a high performance computer cluster belonging to The Victorian Partnership for Advanced Computing (VPAC). We thank the anonymous reviewers for their constructive comments.

\section{References}

Archetti, C., Speranza, M., and Vigo, D. 2013. Vehicle routing problems with profits. Working Papers WPDEM 2013/3, Department of Ecomonics and Management, University of Brescia.

Australasian Fire and Emergency Services Authorities Council 2013. The Australasian Inter-service Incident Management System. AFAC, East Melbourne, 4 edition.

Christofides, N., Mingozzi, A., and Toth, P. 1979. The vehicle routing problem. In Combinatorial Optimizations. Edited by N. Christofides, R. Mingozzi, P. Toth, and C. Sandi. John Wiley \& Sons, New York.

CSIRO 2009. Q\&A: Victorian bushfires [online]. Available from http://www.csiro.au/en/ Outcomes/Safeguarding-Australia/Victorian-Bushfires-QA [accessed 12 May 2014].

Donovan, G.H., and Rideout, D.B. 2003. An integer programming model to optimize resource allocation for wildfire containment. For. Sci. 49(2): 331-335.

Feillet, D., Dejax, P., and Gendreau, M. 2005. Traveling salesman problems with profits. Transportation Science 39(2): 188-205. doi:10.1287/trsc.1030.0079.

Haight, R.G., and Fried, J.S. 2007. Deploying wildland fire suppression resources with a scenariobased standard response model. INFOR: Information Systems and Operational Research 45(1): 31-39. doi:10.3138/infor.45.1.31.

Homchaudhuri, B., Kumar, M., and Cohen, K. 2013. Genetic Algorithm Based SimulationOptimization for Fighting Wildfires. International Journal of Computational Methods. 10(06): 1350035. doi:10.1142/S0219876213500357. 
$\mathrm{Hu}, \mathrm{X}$. and Ntaimo, L. 2009. Integrated simulation and optimization for wildfire containment. ACM Transactions on Modeling and Computer Simulation 19(4): 1-29. doi:10.1145/1596519.1596524.

Hyde, M. 2013. 2013 Tasmanian Bushfires Inquiry. Technical report, Office of Security and Emergency Management, Tasmanian Goverment.

ICS Canada 2012. Incident command system operational description [online]. Available from http://www.icscanada.ca/ [accessed 9 May 2014].

Lee, Y., Fried, J.S., Albers, H.J., and Haight, R.G. 2013. Deploying initial attack resources for wildfire suppression: spatial coordination, budget constraints, and capacity constraints. Can. J. For. Res. 43(1): 56-65. doi:10.1139/cjfr-2011-0433.

Martell, D.L. 1982. A review of operational research studies in forest fire management. Can. J. For. Res. 12(2): 119-140. doi:10.1139/x82-020.

Martell, D.L. 2007. Forest fire management: current practices and new challenges for operational researchers. In Handbook of operations research in natural resources. Edited by A. Weintraub, C. Romero, T. Bjrndal, R. Epstein, and J. Miranda. Springer Science plus Business Media, New York. pp. 489-509.

Martell, D.L., Gunn, E.A., and Weintraub, A. 1998. Forest management challenges for operational researchers. European Journal of Operational Research 104(1): 1-17. doi:10.1016/ S0377-2217(97)00329-9.

Martin-Fernández, S., Martínez-Falero, E., and Pérez-González, J. M. 2002. Optimization of the resources management in fighting wildfires. Environmental Management. 30(3): 352-64. doi: 10.1007/s00267-002-2430-3.

McLennan, J., Holgate, A.M., Omodei, M.M., and Wearing, A.J. 2006. Decision Making Effectiveness in Wildfire Incident Management Teams. Journal of Contingencies and Crisis Management 14(1): 27-37. doi:10.1111/j.1468-5973.2006.00478.x.

Mees, R., Strauss, D., and Chase, R. 1994. Minimizing the cost of wildland fire suppression: a model with uncertainty in predicted flame length and fire-line width produced. Can. J. For. Res. 24(6), 1253-1259. doi:10.1139/x94-164.

Minas, J.P., Hearne, J.W., and Handmer, J.W. 2012. A review of operations research methods applicable to wildfire management. Int. J. Wildland Fire, 21(3): 189-196. doi:10.1071/WF10129.

Ntaimo, L., Gallego Arrubla, J.A., Stripling, C., Young, J., and Spencer, T. 2012. A stochastic programming standard response model for wildfire initial attack planning. Can. J. For. Res. 42(6): 987-1001. doi:10.1139/x2012-032.

Omodei, M.M., McLennan, J., Elliott, G.C., Wearing, A.J., and Clancy, J.M. 2005a. More Is Better? A bias toward overuse of resources in naturalistic decision-making settings. In How professionals make decisions. Edited by H. Montgomery, R. Lipshitz, and B. Brehmer. Lawrence Erlbaum, Mahwah, NJ. pp 29-41. 
Omodei, M.M., Mclennan, J., Reynolds, C. 2005b. Identifying why even well-trained firefighters make unsafe decisions: a human factors interview protocol. In Proceedings of the Eight International Wildland Fire Safety Summit, Missoula, MT, 26-28 April 2005. Edited by B. Butler and M. Alexander. International Association of Wildland Fire, Hot Springs, SD.

Pappis, C.P. and Rachaniotis, N.P. 2010a. Scheduling a single fire fighting resource with deteriorating fire suppression times and set-up times. Operational Research 10(1): 27-42. doi: 10.1007/s12351-009-0038-6.

Pappis, C.P. and Rachaniotis, N.P. 2010b. Scheduling in a multi-processor environment withdeteriorating job processing times and decreasing values: the case of forest fires. Journal of Heuristics 16(4): 617-632. doi:10.1007/s10732-009-9110-x.

Sullivan, A.L. 2009a. Wildland surface fire spread modelling, 1990-2007. 1: Physical and quasiphysical models. Int. J. Wildland Fire, 18(4): 349-368. doi:10.1071/WF06143.

Sullivan, A.L. 2009b. Wildland surface fire spread modelling, 1990-2007. 2: Empirical and quasiempirical models. Int. J. Wildland Fire, 18(4): 369-386. doi:10.1071/WF06142.

Sullivan, A.L. 2009c. Wildland surface fire spread modelling, 1990-2007. 3: Simulation and mathematical analogue models. Int. J. Wildland Fire, 18(4): 387-403. doi:10.1071/WF06144.

US Department of Homeland Security 2008. National Incident Management System. US Government, Washington, DC.

Van der Merwe, M., Minas, J.P., Ozlen, M., and Hearne, J.W. 2014. The cooperative orienteering problem with time windows. Optimization Online, April 2014. Available from http://www. optimization-online.org/DB_HTML/2014/04/4316.html [accessed 9 May 2014].

Vansteenwegen, P., Souffriau, W., and Van Oudheusden, D. 2011. The orienteering problem: A survey. European Journal of Operational Research. 209(1): 1-10. doi:10.1016/j.ejor.2010.03.045. 